\title{
The IL-12R $\beta 2$ gene functions as a tumor suppressor in human B cell malignancies
}

\author{
Irma Airoldi, ${ }^{1}$ Emma Di Carlo, ${ }^{2}$ Barbara Banelli, ${ }^{3}$ Lidia Moserle, ${ }^{4}$ Claudia Cocco, ${ }^{1}$ Annalisa Pezzolo, ${ }^{1}$ \\ Carlo Sorrentino, ${ }^{2}$ Edoardo Rossi,, 5 Massimo Romani, ${ }^{3}$ Alberto Amadori,, ${ }^{4}$ and Vito Pistoia ${ }^{1}$
}

\begin{abstract}
'Laboratory of Oncology, G. Gaslini Institute, Genoa, Italy. ${ }^{2}$ Department of Oncology and Neurosciences, G. D’Annunzio University, Chieti, Italy. ${ }^{3}$ Laboratory of Tumor Genetics, Istituto Nazionale per la Ricerca sul Cancro, Genoa, Italy. ${ }^{4}$ Department of Oncology and Surgical Sciences, University of Padua, Padua, Italy. ${ }^{5}$ Department of Hematology, Azienda Ospedaliera S. Martino, Genoa, Italy.
\end{abstract}

\begin{abstract}
The $I L-12 R \beta 2$ gene is expressed in human mature B cell subsets but not in transformed B cell lines. Silencing of this gene may be advantageous to neoplastic $B$ cells. Our objective was to investigate the mechanism(s) and the functional consequence(s) of $I L-12 R \beta 2$ gene silencing in primary $\mathrm{B}$ cell tumors and transformed $\mathrm{B}$ cell lines. Purified tumor cells from 41 patients with different chronic B cell lymphoproliferative disorders, representing the counterparts of the major mature human B cell subsets, tested negative for $I L-12 R \beta 2$ gene expression. Hypermethylation of a CPG island in the noncoding exon 1 was associated with silencing of this gene in malignant B cells. Treatment with the DNA methyltransferase inhibitor 5-Aza-2'-deoxycytidine restored IL-12R $\beta 2$ mRNA expression in primary neoplastic B cells that underwent apoptosis following exposure to human recombinant IL-12 (hrIL-12). hrIL-12 inhibited proliferation and increased the apoptotic rate of $I L-12 R \beta 2$-transfected B cell lines in vitro. Finally, hrIL-12 strongly reduced the tumorigenicity of $I L-12 R \beta 2-$ transfected Burkitt lymphoma RAJI cells in SCID-NOD mice through antiproliferative and proapoptotic effects, coupled with neoangiogenesis inhibition related to human IFN- $\gamma$-independent induction of hMig/ CXCL9. The IL-12R $\beta 2$ gene acts as tumor suppressor in chronic B cell malignancies, and IL-12 exerts direct antitumor effects on $I L-12 R \beta 2$-expressing neoplastic B cells.
\end{abstract}

\section{Introduction}

IL-12 is a heterodimeric cytokine bridging innate resistance and antigen-specific adaptive immunity (1). IL-12 is produced predominantly by professional APCs and serves as an important regulator of T cell and NK cell function (2,3). IL-12 has a powerful antitumor activity related to both IFN- $\gamma$-dependent and -independent mechanisms (4-9).

Numerous effects of IL-12 on human B cells from normal individuals have been reported, such as induction of proliferation and differentiation to Ig-secreting cells (10), upregulation of IL-18R (11), and induction of IFN- $\gamma$ mRNA and protein (11-13).

The biological functions of human IL-12 are mediated by the heterodimeric IL-12R composed of two subunits, the $\beta 1$ and the $\beta 2$ chains, conferring high-affinity binding of and responsiveness to IL-12 $(14,15)$. The $\beta 1$ chain participates in the formation of the IL-23 receptor, whereas the $\beta 2$ chain is an unique component of IL-12R (1). The IL-12R $\beta 2$ gene maps on chromosome $1 \mathrm{p} 31.2$ and is composed of 16 introns and 16 exons, the first of which is not transcribed (15).

We have previously shown that human lymphoblastoid B cell lines (LCLs) and Burkitt lymphoma (BL) cell lines express constitutively IL-12R $\beta 1$ mRNA but lack IL-12R $\beta 2$ mRNA (16). In contrast, normal human naive, germinal center (GC), and memory B cells express constitutively the transcripts of both $I L-12 R$ genes (11). These find-

Nonstandard abbreviations used: B chronic lymphocytic leukemia (B-CLL); Burkitt lymphoma (BL); follicular lymphoma (FL); follicular mantle (FM); germinal center (GC); green fluorescent protein (GFP); human IFN- $\gamma$ (hIFN- $\gamma$ ); human recombinant IL-12 (hrIL-12); lymphoblastoid cell line (LCL); mantle cell lymphoma (MCL); marginal zone lymphoma (MZL); methylation-specific PCR (MSP); phycoerythrin (PE); subepithelial (SE).

Conflict of interest: The authors have declared that no conflict of interest exists.

Citation for this article: J. Clin. Invest. 113:1651-1659 (2004).

doi:10.1172/JCI200420303. ings led us to speculate that malignant B cells could benefit from the silencing of the $I L-12 R \beta 2$ gene.

Here we first investigated $I L-12 R$ gene expression in primary tumor cells from patients with different chronic B cell lymphoproliferative disorders representing the postulated counterparts of the major mature $B$ cell subsets, as no information was available on this issue. IL-12R $\beta 2$ mRNA expression was not detected in any sample. Second, we studied the mechanism(s) involved in and the functional consequences of the silencing of this gene in human primary neoplastic $B$ cells, as well as in transformed B cell lines. Our findings support the conclusion that the $I L-12 R \beta 2$ gene functions as tumor suppressor in a wide spectrum of human $B$ cell malignancies.

\section{Results}

Expression of IL-12R in normal tonsil $B$ cell subsets and in malignant $B$ cells from different human chronic lymphoproliferative disorders. Figure 1A shows one experiment, representative of the ten performed with identical results, in which the IL-12R $\beta 1$ and $\beta 2$ transcripts were detected in naive, GC, and memory $B$ cells isolated from tonsil, consistently with a previous study (11). As is apparent, these B cell fractions expressed CD19, but not CD3 $\gamma$, CD56, or CD68, mRNA.

In subsequent studies, frozen tonsil tissue sections were stained sequentially with phycoerythrin-conjugated (PE-conjugated) anti-IL-12R $\beta 2$ and FITC-conjugated CD19 mAb's or isotype and fluorochrome matched control mAb's and then with DAPI. Images were acquired using either a filter selective for fluorochrome (Figure 1, C, G, K and D, H, L) or a triple-band - i.e., DAPI, PE, and FITC-specific - filter (Figure 1, E, I, M and F, J, N).

Figure 1B shows a DAPI staining with the follicular mantle (FM), the GC, and the subepithelial (SE) area in boxes. Figure 1, C, G, K and D, H, L, shows staining with CD19 (green) or anti-IL$12 \mathrm{R} \beta 2$ (red) mAb's, respectively. As is apparent, the large majority of cells positioned in the FM, GC, and SE areas expressed CD19 